\title{
Three-dimensional simulation of facet formation and the coupled heat flow and segregation in Bridgman growth of oxide crystals
}

\author{
C.W. Lan*, C.Y. Tu \\ Department of Chemical Engineering, National Taiwan University, Taipei 10617, Taiwan, ROC \\ Received 3 April 2001; accepted 6 July 2001 \\ Communicated by D.T.J. Hurle
}

\begin{abstract}
Three-dimensional (3D) simulation of facet formation and its coupled heat flow and segregation in the Bridgman growth of oxide crystals is presented. The growth of YAG crystals in the [1 11 l] direction, with $\left\{\begin{array}{lll}2 & 1\end{array}\right\}$ facets, is taken as an example to illustrate the finite volume treatment of the interfacial kinetics involving supercooling and crystallographic constrains. The simulation successfully reveals experimental observations, and the growth conditions for reducing faceting are consistent with growth experience in general. Increasing thermal gradients or reducing interface convexity reduces the faceting area. In addition, 3D convection and dopant segregation due to faceting are illustrated. The simulation also allows a different segregation coefficient at the facets, so that the abnormal segregation, such as the dark core, due to faceting can be simulated as well. (C) 2001 Elsevier Science B.V. All rights reserved.
\end{abstract}

PACS: 44.25. +f; 47.27.Te; 81.10.Fq; 02.60.c6; 02.70.Fj

Keywords: A1. 3D simulation; A1. Convection; A1. Segregation; A2. Bridgman growth; B2. Oxide crystals

\section{Introduction}

Facet formation during the growth of oxide crystals is an important phenomenon in crystal growth [1-3]. For example, in the growth of garnet crystals, such as YAG and GGG, in the $\langle 1111\rangle$ direction, faceting at $\{211\}$ crystallographic planes is typical $[2,4,5]$. Faceting can affect the crystal quality in several ways. The most common ones are the stress concentration at the singular

\footnotetext{
*Corresponding author. Tel./fax: +886-2-2363-3917.

E-mail address: lan@ruby.che.ntu.edu.tw (C.W. Lan).
}

edges and the severe segregation due to different segregation coefficients in the facets. The dark core in the growth of $\mathrm{Bi}_{12} \mathrm{Si}_{20} \mathrm{O}$ (BSO) is also an example of the ion segregation due to faceting [6]. Therefore, the control of facet formation is an important task in the improvement of crystal quality. Growing the crystal in a proper direction is an effective way to reduce faceting. However, if a growth direction is chosen, the faceting is in general controlled by the interface morphology and thermal gradients. In such a case, the optimization of heat flow is crucial and computer simulation may play an important role in tuning 
growth conditions. However, computer simulation of faceting involving heat and mass transfer and melt flow, as well as the unknown interface shape, remains a great challenge in crystal growth modeling [7]. In addition to the interfacial kinetics, the problem is truly three dimensional (3D) and the free interface is subjected to the constrains of supercooling and crystallographic orientations. The first attempt to simulate the faceting coupled with heat transfer in oxide growth was by Brandon et al. [8,9] using a finite element approach. However, their simulations were restricted to 2D; for example, the growth is in the [lllll direction with a $\left\{\begin{array}{lll}1 & 1 & 1\end{array}\right\}$ facet. Therefore, the 3D faceting during crystal growth cannot be simulated by their codes. As pointed out by Hurle [7], faceting is important in oxide growth and a realistic simulation should take it into account. Therefore, in this report, we present, for the first time, a 3D simulation of the faceting phenomena in the Bridgman growth of oxide crystals. In addition to the self-consistent simulation of the interface morphology, the melt flow and heat and mass transfer are considered simultaneously.

Based on this model, in this report we reveal a typical faceting observed in experiments through numerical simulation. The control of faceting will also be discussed. In addition, the interaction of faceting with the melt flow and dopant segregation will be illustrated. In the next section, the model and its numerical simulation are briefly described. Section 3 is devoted to the results and discussion, followed by conclusions in Section 4.

\section{Model description and numerical solution}

A generic Bridgman crystal growth configuration is shown Fig. 1a. Due to the faceting at the interface (or imperfect growth environment), the problem is $3 \mathrm{D}$ and thus described by Cartesian coordinates $(x, y, z)$. The ambient thermal distribution $T_{\mathrm{a}}(x, y, z)$ is assumed to be known. To focus on the facet formation, we have adopted a pseudosteady state approximation in this report. The magnitude of the ampoule pulling speed $U_{\mathrm{amp}}$ is assumed to be the same as the axial growth rate .
This assumption is applied to the dopant calculation as well by using an artificial inlet condition at $x=L[10]$.

The flow, temperature, and dopant fields, as well as the melt/crystal interface, are also represented in the Cartesian coordinates $(x, y, z)$. Furthermore, it is also assumed that the dopant concentration is so low that its effects on the flow and liquidus temperature can be neglected. The melt is also assumed to be opaque [11]. Moreover, the dimensionless variables are defined by scaling length with the crystal diameter $D_{\mathrm{c}}$, velocity with $\alpha_{\mathrm{m}} / D_{\mathrm{c}}$, pressure with $\rho_{\mathrm{m}} \alpha_{\mathrm{m}}^{2} / D_{\mathrm{c}}^{2}$, and dopant concentration with its average concentration $C_{0}$ in the crystal, where $\rho_{\mathrm{m}}$ is the melt density and $\alpha_{\mathrm{m}}$ the thermal diffusivity. The dimensionless temperature is scaled by the melting point $T_{\mathrm{m}}$. For the convenience of discussion, the variables used in the equations are all dimensionless unless otherwise stated. The pseudo-steady governing equations describing convection and heat and dopant transport in the melt (m) are as follows:

$$
\begin{aligned}
& \nabla \cdot \boldsymbol{v}=0, \\
& \boldsymbol{v} \cdot \nabla \boldsymbol{v}=-\nabla P+\operatorname{Pr} \nabla^{2} \boldsymbol{v}-\operatorname{Pr} \operatorname{Ra}_{\mathrm{T}}(T-1) \boldsymbol{e}_{\mathrm{g}}, \\
& \boldsymbol{v} \cdot \nabla T=\nabla^{2} T, \\
& \boldsymbol{v} \cdot \nabla C=\frac{\operatorname{Pr}}{\mathrm{Sc}} \nabla^{2} C,
\end{aligned}
$$

where $\boldsymbol{v}, P, T$, and $C$ are the dimensionless velocity, pressure, temperature, and dopant concentration, respectively. $\operatorname{Pr}$ is the Prandtl number $\left(\operatorname{Pr} \equiv v_{\mathrm{m}} / \alpha_{\mathrm{m}}\right)$ and $\mathrm{Sc}$ the Schmidt number $\left(\mathrm{Sc} \equiv v_{\mathrm{m}} / D\right)$, where $v_{\mathrm{m}}$ is the kinematic viscosity and $D$ the dopant diffusivity in the melt. In Eq. (2), the thermal Rayleigh number $\mathrm{Ra}_{\mathrm{T}}$ in the source term is defined as follows:

$\mathrm{Ra}_{\mathrm{T}} \equiv \frac{g \beta_{\mathrm{T}} T_{\mathrm{m}} D_{\mathrm{c}}^{3}}{\alpha_{\mathrm{m}} v_{\mathrm{m}}}$,

where $g$ is the gravitational acceleration and $\beta_{\mathrm{T}}$ the thermal expansion coefficient. Furthermore, the gravity unit vector $\boldsymbol{e}_{\mathrm{g}}$ is defined as

$\boldsymbol{e}_{\mathrm{g}}=-\cos (\gamma) \boldsymbol{e}_{x}-\sin (\gamma) \boldsymbol{e}_{y}$,

where $\gamma$ is the tilt angle from the growth axis. 


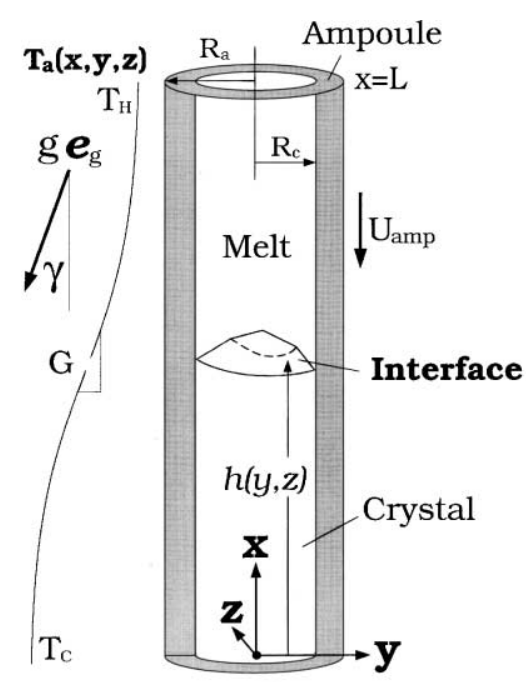

(a)

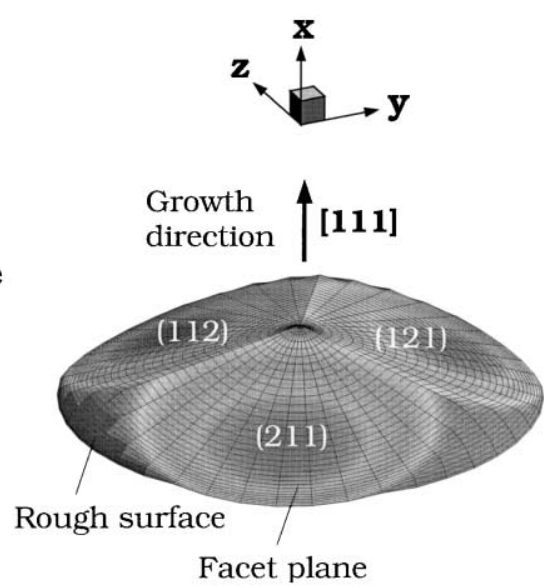

(b)

Fig. 1. (a) Schematic sketch of a Bridgman oxide growth; (b) a sample mesh for the interface with $\{211\}$ facets; the growth direction is in $\left[\begin{array}{lll}1 & 1 & 1\end{array}\right]$.

In the crystal (c) and the ampoule (a), only heat transfer needs to be considered. However, for most oxides, except those highly doped, the crystal is semitransparent. Accordingly, internal radiation cannot be ignored. Detailed calculation of internal radiation is complicated and computing-extensive. Brandon and Derby [11] have considered that for a $2 \mathrm{D}$ problem in a rigorous manner, which requires the solution of differential-integral equations. For a highly transparent crystal, the interface convexity could be much larger than the opaque one. However, in this report, to reduce the computational cost and to concentrate the discussion on faceting, the internal radiation inside the crystal is treated by the Rosseland diffusion approximation $[12,13]$ using the so-called effective thermal conductivity. Therefore, the heat transfer in the solid can still be represented as usual:

$\mathrm{Pe}_{i} \boldsymbol{e}_{x} \cdot \nabla T=\nabla \kappa_{i}(T) \nabla T \quad(i=\mathrm{c}, \mathrm{a})$,

where $\mathrm{Pe}_{i} \equiv \rho_{i} C_{p_{i}} U_{\mathrm{amp}} D_{\mathrm{c}} / k_{\mathrm{m}}$ is the Peclect number and $\kappa_{i}(T) \equiv k_{i}(T) / k_{\mathrm{m}}$ the dimensionless thermal conductivity of crystal or ampoule; $k_{\mathrm{m}}$ is the thermal conductivity of the melt at $T_{\mathrm{m}}$. Also, $\rho_{i}$, $C_{p_{i}}$ and $k_{i}$ are the density, specific heat, and thermal conductivity of the phase $i(i=\mathrm{c}$ or a), respectively. Notably, the Rosseland approxima- tion treats the crystal thermal conductivity (in the dimensional form) as follows [14]:

$k_{\mathrm{c}}=k_{\mathrm{c}}^{\mathrm{cond}}+\frac{16 n^{2} \sigma T^{3}}{3 a_{\mathrm{R}}}$,

where $k_{\mathrm{c}}^{\text {cond }}$ is the contribution due to conduction and the second term is due to radiation. Also, $n$ is the refractive index, $\sigma$ the Stefan-Boltzmann constant, and $a_{\mathrm{R}}$ the Rosseland mean absorption coefficient. As shown, the internal radiation enhances the heat transfer (cooling effect) in the crystal. Due to the increase of the crystal conductivity, the interface convexity is increased with the decreasing absorption coefficient $a_{\mathrm{R}}$. Nevertheless, because the Rosseland approximation is not accurate near the boundary and is valid only for optically thick materials, we do not anticipate a satisfactory solution from there for small $a_{\mathrm{R}}$. In fact, for a consistent formulation, a jump boundary condition, such as the Deissler's condition [14], is needed. Nevertheless, in a recent study by Vizman et al. [12,13], this treatment seems to be reasonable for oxide growth. Therefore, we have adopted this approach to evaluate the effect of internal radiation. Although we do not want to focus on this topic, one should keep in 
mind that a much more rigorous treatment is required for an accurate prediction.

The no-slip condition is used for the melt velocity on solid boundaries. When the pseudosteady state is assumed, the upper open boundary is considered as an artificial boundary [10], and its velocity boundary condition is kept the same. The thermal and solutal boundary conditions at the melt/solid interfaces are set by the heat and mass flux balances. For example, at the growth front

$$
\begin{aligned}
& \left.\boldsymbol{n} \cdot \nabla T\right|_{\mathrm{m}}-\left.\boldsymbol{n} \cdot \kappa_{\mathrm{c}} \nabla T\right|_{\mathrm{c}}-\gamma_{\mathrm{c}} \operatorname{Pe}_{\mathrm{m}} \operatorname{St}\left(\boldsymbol{e}_{x} \cdot \boldsymbol{n}\right)=0, \\
& -\left.\boldsymbol{n} \cdot \nabla C\right|_{\mathrm{m}}+\gamma_{\mathrm{c}} \operatorname{Pe}_{\mathrm{m}} \frac{\mathrm{Sc}}{\operatorname{Pr}}(1-K) C\left(\boldsymbol{e}_{x} \cdot \boldsymbol{n}\right)=0,
\end{aligned}
$$

where $\boldsymbol{n}$ is the unit normal vector at the growth interface pointing to the melt. The Stefan number $\mathrm{St} \equiv \Delta H /\left(C_{p_{\mathrm{m}}} T_{\mathrm{m}}\right)$ scales the heat of fusion $(\Delta H)$ released during solidification to the sensible heat in the melt; $\gamma_{\mathrm{c}}=\rho_{\mathrm{c}} / \rho_{\mathrm{m}}$. The equilibrium segregation coefficient $K$ of the solute is according to the phase diagram; $K \equiv C_{\mathrm{c}} / C$ at the growth interface, where $C_{\mathrm{c}}$ is the dopant concentration in the crystal. However, $K$ is allowed to be different at different crystallographic planes here.

The heat exchange between the ampoule and the furnace is by both radiation and convection according to the energy balance along the ampoule surface:

$$
-\left.\boldsymbol{n} \cdot \kappa_{\mathrm{a}} \nabla T\right|_{\mathrm{a}}=\operatorname{Bi}\left(T-T_{\mathrm{a}}\right)+\operatorname{Rad}\left(T^{4}-T_{\mathrm{a}}^{4}\right),
$$

where $\vec{n}$ is the unit normal vector on the ampoule surface pointing outwards, $\mathrm{Bi} \equiv h D_{\mathrm{c}} / k_{\mathrm{m}}$ the Biot number, and $\operatorname{Rad} \equiv \sigma \varepsilon_{\mathrm{a}} T_{\mathrm{m}}^{3} D_{\mathrm{c}} / k_{\mathrm{m}}$ the Radiation number; $\varepsilon_{\mathrm{a}}$ is the surface emissivity of the ampoule. For simplicity, the effective furnace temperature $T_{\mathrm{a}}$ is assumed to be a hyperbolic tangent function for most cases:

$$
T_{\mathrm{a}}=T_{\mathrm{C}}+0.5\left(T_{\mathrm{H}}-T_{\mathrm{C}}\right)\left[1+\frac{\tanh (d(x-L / 2))}{\tanh (0.5 d L)}\right],
$$

where $T_{\mathrm{C}}$ and $T_{\mathrm{H}}$ are the cold- and hot-zone temperatures, $d$ is related to the length of the adiabatic zone, and $L$ is the sample length. Temperature at the top and bottom surfaces is set to be the furnace temperature there. For the study of thermal gradients, a linear heating profile is also used. Again, for both cases, $T_{\mathrm{m}}$ is located at $x=0.5 L$.

Also, the temperature at the melt/crystal interface for a rough surface is assumed to be at the equilibrium liquidus temperature of the material; the supercooling at the rough surface is neglected. For a convex interface, this is a reasonable assumption because the supercooling at the singular faces (facets) is much larger. On the contrary, for a concave interface, the $2 \mathrm{D}$ screw growth mechanism shows no superheating in the facet. In such a case, the supercooling at the rough surface becomes important [5]. Due to the internal radiation in the crystal, the interface appears to be convex for most of the cases. For a convex interface, if a facet exists, the interface is defined by its crystallographic plane and the highest supercooling in the plane is equal to the kinetic supercooling $\Delta T_{\text {kin. }}$. Therefore, in the facet the lowest temperature is defined by

$T=T_{\mathrm{m}}-\Delta T_{\text {kin }}$.

The supercooling $\Delta T_{\text {kin }}$ is a kinetic data and it also depends on the mechanisms of facet formation. In general, for a facet plane, it is related to the growth rate. The simplest form may be taken as the following:

$\Delta T_{\mathrm{kin}}=\beta U_{\mathrm{n}}^{c}=\beta\left[\left|U_{\mathrm{amp}}\right|\left(\boldsymbol{n} \cdot \boldsymbol{e}_{x}\right)\right]^{c}$,

where $\beta$ is the kinetic coefficient, $U_{\mathrm{n}}$ the normal growth rate at the interface, and $c$ is a constant depending on the mechanisms. For example, if the facet is formed by a screw dislocation (growth), $c$ is about 0.5 . For a given growth rate, $\Delta T_{\text {kin }}$ also depends on the interface shape. However, for a given facet, $\left(\boldsymbol{n} \cdot \boldsymbol{e}_{x}\right)$ is fixed. Therefore, once the steady state growth rate is achieved, for a given facet, $\Delta T_{\text {kin }}$ can be used as an input parameter (from the kinetic data). Therefore, for the $\left\{\begin{array}{lll}2 & 1 & 1\end{array}\right\}$ facet, the maximum supercooling is known if the growth rate is specified. In fact, our approach for treating the faceting is similar to the one proposed by Brice [1] and Voronkov [15], which is based on simple geometries relating the facet size $b$ (diameter) by

$b=\sqrt{8 \Delta T_{\text {kin }} R / G}$, 
where $R$ is the radius of curvature of the melting point isotherm and $G$ the local thermal gradient. However, obtaining $R$ and $G$ a priori is not possible without detailed heat flow simulation. Therefore, in the present report, their concept for facet formation is adopted, but the interface shape and the local thermal gradients are coupled with the heat flow calculation. For a time-dependent simulation, the actual interface speed needs to be used, and one can put the kinetic constants $(\beta$ and $c$ ) into the model. As such, the local supercooling and facet formation are also time dependent.

Once the kinetic information is known, to implement the facet calculation is straightforward. Take the growth in the [lllll 111 direction with $\left\{\begin{array}{lll}2 & 1 & 1\end{array}\right\}$ facets as an example. We first align the [ $\left.\begin{array}{lll}1 & 1 & 1\end{array}\right]$ direction with the $x$-axis. By a simple rotation transformation, [ $\left.\begin{array}{lll}1 & 0 & 1\end{array}\right]$ is in the $z$-direction, while $\left[\begin{array}{lll}1 & 2 & 1\end{array}\right]$ is in the $y$-direction. Furthermore, the plane normal of the three $\{211\}$ facets can be easily obtained as well, as shown in Fig. 1 b: they are $4 \boldsymbol{e}_{x} / \sqrt{3}-\boldsymbol{e}_{y} / \sqrt{6}+\boldsymbol{e}_{z} / \sqrt{2}, \quad 4 \boldsymbol{e}_{x} / \sqrt{3}+\boldsymbol{e}_{y} / \sqrt{6}+$ $\boldsymbol{e}_{z} / \sqrt{2}$, and $4 \boldsymbol{e}_{x} / \sqrt{3}-\boldsymbol{e}_{y} / \sqrt{6}-\boldsymbol{e}_{z} / \sqrt{2}$, respec-

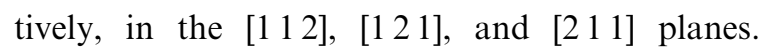
Because at the growth axis, $y=0$ and $z=0$, one can use a plane equation, i.e., $f(x, y, z)=x+B y+$ $C z+D=0$, to define each facet, where $B, C$, and $D$ are constants; $-D=x_{0}$ is the intersection of the plane with the $x$-axis. The plane constants $B$ and $C$ can be easily calculated from the normal vector $(\nabla f)$; e.g., for the $\left[\begin{array}{ll}1 & 1\end{array}\right]$ plane, $B=1 /(4 \sqrt{2})$ and $C=\sqrt{3} /(4 \sqrt{2})$.

During calculation, the interface update is straightforward. Once the energy and momentum equations are solved, the location of $T_{\mathrm{m}}$ is found first for the rough surface, where the thermal boundary condition, Eq. (8), is used for the energy equation. Then the interface is cut by the facet plane; at the beginning one can use the melt height at $y=0$ and $z=0$ to start with. If the new interface (the cut plane) is lower than the rough one, the interface is updated. Based the new interface shape, remeshing is performed and the temperature as well as other variables, are then updated, and the highest supercooling in each facet is found. If the supercooling is higher than the kinetic one, the plane is moved upward by increasing $x_{0}$. This procedure continues until all the supercooling constraints are satisfied and all the facets are tested. Because the interface is defined by the nodes as shown in Fig. 1a, the vertices of the mesh are then interpolated from the node values. Both nodes and vertices are used for remeshing. This procedure is robust, and no special treatment is needed after finding the interface shape. It needs to be pointed out that the present scheme is mainly based on the geometric reasoning due to Brice [1] and Voronkov [15]. It is slightly different from the scheme proposed by Brandon et al. [8,9]. In their computation, they introduced a kinetic coefficient $\beta$, which is a function of crystallographic orientation, to smooth out the sharp edge between the facet and rough surfaces. Although their approach has its physical reasoning from the microscopic point of view, the treatment is still numerical. Indeed, for macroscopic modeling, the capillary length scale is much less than the mesh size used in calculation. Therefore, the heat flow and thus the mass transport will not be affected much by the rounded edges due to interfacial energy. Even for the stress calculation at the singular edges formed by the touch of two facets, their approach may not provide a quantitative radius of curvature there. On the other hand, the interface and facets calculated by the present approach are adequate enough for further estimation of the local curvature and stress calculations if a local model can be introduced.

The above governing equations and their associated boundary conditions are solved by an efficient finite volume method (FVM) scheme using multigrid acceleration [16] for the free boundary problem. Two levels of grids are used in the calculation. In the first level, there are $16 \times$ $21 \times 30$ (in the radial, angular, and axial directions, respectively) finite volumes in the melt and $16 \times 21 \times 15$ in the crystal, and $5 \times 21 \times 45$ in the ampoule. The second level doubles the finite volumes in each direction, i.e., $32 \times 42 \times 60$ cells in the melt. The calculations are performed in personal computers (PIII/800 MHz CPU with 512M SRAM), and one calculation takes about one hour of CPU time. For all cases, the results based on the coarse and fine grids are about the 
same. Detailed description of the numerical method can be found elsewhere [16].

\section{Results and discussion}

In this study, we have taken the Bridgman growth of YAG crystals as an example. The growth is in the [1 111 ] direction, and the typical faceting has been found to be the $\left\{\begin{array}{lll}2 & 1 & 1\end{array}\right\}$ planes [5]; the angle between [2 111$]$ and [ 1111$]$ is about $19.47^{\circ}$. Sometimes, $\left\{\begin{array}{lll}1 & 1 & 0\end{array}\right\}$ facets can also be found in the experiments, which occurs usually near the edge of the interface due to the larger critical angle being about $35.26^{\circ}$. The data and parameters used in the simulation are summarized in Table 1. The melt viscosity is the same as the one used by Brandon and Derby [11] and this value is also consistent with the recent measurement [17]; however, this value is a bit higher than other oxides. Therefore, the convection is too weak to affect much the isotherms and thus the interface morphology. Accordingly, in this report, the role of convection intensity is illustrated by reducing the viscosity or tilting the ampoule. Furthermore, the Rosseland mean absorption coefficient $a_{\mathrm{R}}$ is also used as an input parameter to consider the effects of internal radiation. Because this approach is very primitive, we do not attempt to use a small value of $a_{\mathrm{R}}$ here.

\subsection{Effect of supercooling}

Fig. 2 shows the effect of supercooling at the facet planes; the flow structures and isotherms at two different planes are presented (in each figure, $y=0$ is on the left and $z=0$ on the right, respectively). The mean absorption coefficient $a_{\mathrm{R}}$ is set to be $5 \mathrm{~cm}^{-1}$. Due to the much larger effective crystal conductivity, the growth interface is quite convex toward the melt [11]. Without supercooling as shown in Fig. 2a, the flow and thermal fields, as well as dopant fields at the interface $\left(C_{\mathrm{c}}=K C\right)$, are axisymmetric. The interface shape (the lowest plot) is axisymmetric as well, and the temperature is uniform at $T_{\mathrm{m}}$. Because the melt convection is very weak, the isotherms are not affected much. With $2^{\circ} \mathrm{C}$ of supercooling, the $\{211\}$ facets, as shown in Fig. 2b, slightly touch one another.
Table 1

Physical properties and some input parameters [11]

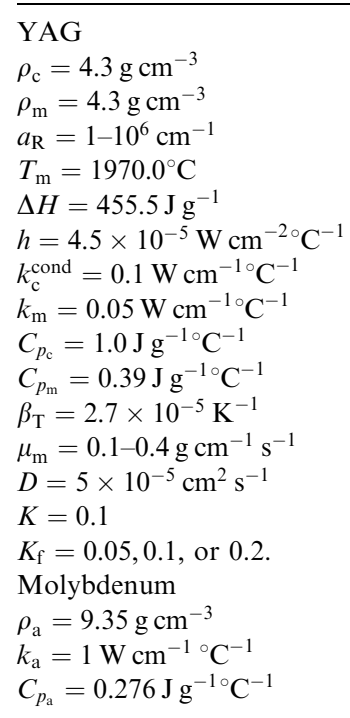

Other input parameters

$L=8 \mathrm{~cm}$

$R_{\mathrm{c}}=0.65 \mathrm{~cm}\left(D_{\mathrm{c}}=2 R_{\mathrm{c}}=1.3 \mathrm{~cm}\right)$

$R_{\mathrm{amp}}=0.967 \mathrm{~cm}$

$T_{\mathrm{H}}=2170^{\circ} \mathrm{C}$

$T_{\mathrm{C}}=1770^{\circ} \mathrm{C}$

$G=25-200^{\circ} \mathrm{C} \mathrm{cm}^{-1}$

$d=0.5 \mathrm{~cm}$

$U_{\mathrm{amp}}=-1 \times 10^{-4} \mathrm{~cm} \mathrm{~s}^{-1}$

$\gamma=0-45^{\circ}$

Dimensionless groups

$\mathrm{Pr}=8 ; \mathrm{Sc}=1860 ; \mathrm{Ra}_{\mathrm{T}}=1.21 \times 10^{5}$

Interestingly, the effect of the asymmetric interface due to faceting on the heat flow seems to be small. The flow and thermal fields are still about symmetric. The interface is no longer at the melting point and the lowest temperature occurs inside the facets.

Furthermore, the segregation at the interface due to faceting increases slightly and shows a three-fold pattern; the rough interface is shaded. At the singular edges, where two facets connect, due to the different surface normals at the two sides of each edge, the iso-concentration lines form a cusp there. This becomes even obvious when the supercoling is further increased to $3.5^{\circ} \mathrm{C}$ in Fig. $2 \mathrm{c}$. Besides the bigger facets, the contiguous facets 


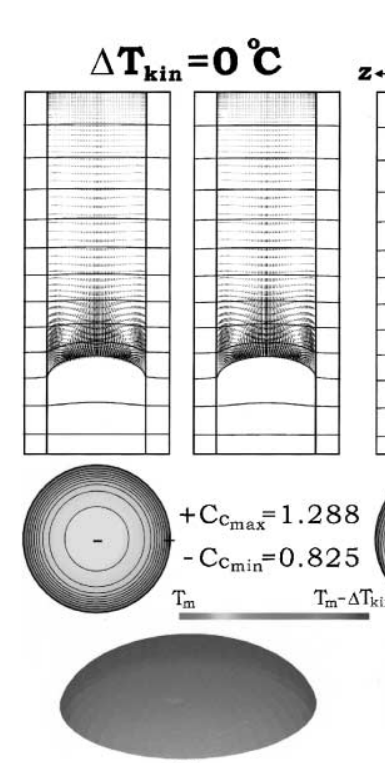

(a)
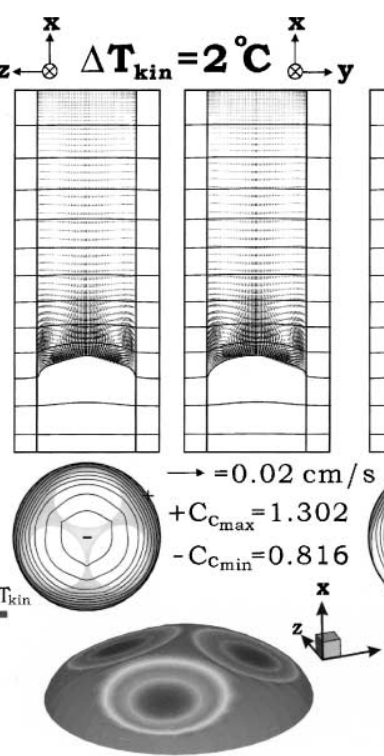

(b)
$\Delta \mathbf{T}_{\text {kin }}=3.5^{\circ} \mathrm{C}$

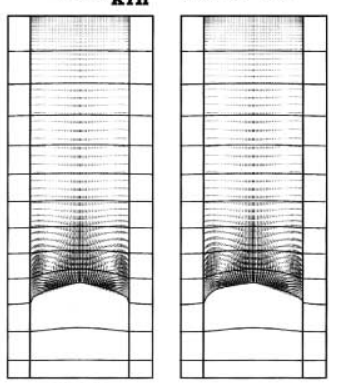

$+\mathrm{C}_{\mathrm{c}_{\max }}=1.285$

$-\mathrm{C}_{\mathrm{c}_{\min }}=0.803$

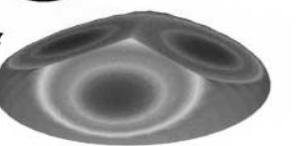

(c)

Fig. 2. Effect of supercooling $\left(\Delta T_{\text {kin }}\right)$ on the flow, thermal and dopant fields, and interface shape: (a) $0^{\circ} \mathrm{C}$; (b) $2^{\circ} \mathrm{C}$; (c) $3.5^{\circ} \mathrm{C}$; $a_{\mathrm{R}}=5 \mathrm{~cm}^{-1}$. The shaded area in concentration plots indicates the rough interface.

touch and form three clear edges; the three facets also join at the center forming a singular point there. These singular edges and point can be the place for stress concentration and defect generation. Hence, the prediction of these edges is critical for improving crystal quality. On the other hand, because the singular edges introduce local elastic stress [18], the local curvature as well as the kinetics of the interface is changed. This may introduce further complication of the local behavior. Nevertheless, as mentioned previously, the length scale of this effect is very small (in the order of micrometers). Therefore, its effect on the macroscopic behavior may be ignored. Besides the larger supercooling, the flow and thermal fields, in general, are not affected much. Again, the effect of faceting on the segregation is clear. Besides the three-fold pattern, the highest dopant concentration appears at the outer edge of the rough surface, as shown by the darkest part of the shaded area. In addition, we have used the same segregation coefficient $(K=0.1)$ for calculation. Therefore, the abnormal segregation due to faceting is not found, and we shall discuss that shortly.
In order to further illustrate the coupling of the thermal field and the faceting, the interface shapes (at two different planes) in the previous calculations are put together for comparison in Fig. 3. As shown, besides the facet cut, the original rough surface remains about the same; larger difference can be found near the edge. Interestingly, although this is a coupled problem, the facet calculation performed afterwards, by cutting the rough surface according to the crystallographic planes with a known supercooling, seems to be a reasonable approximation. Nevertheless, its effects on the heat flow and segregation cannot be overlooked. Even though the global flow structure is not much affected by the faceting, for the local flow, if it is magnified to the size shown in Fig. 3, one can still observe its asymmetry, and indeed this causes the difference in the segregation patterns shown in Fig. 2.

\subsection{Effect of internal radiation}

It is well known that the internal radiation inside the crystal enhances the cooling leading to a more convex interface [11]. The interface convexity 


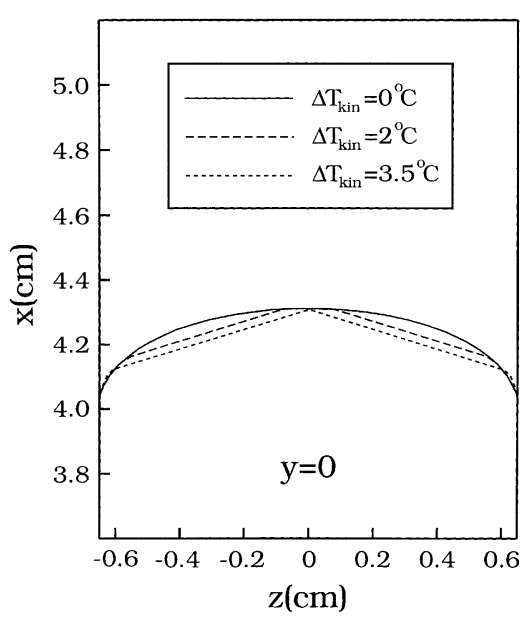

(a)

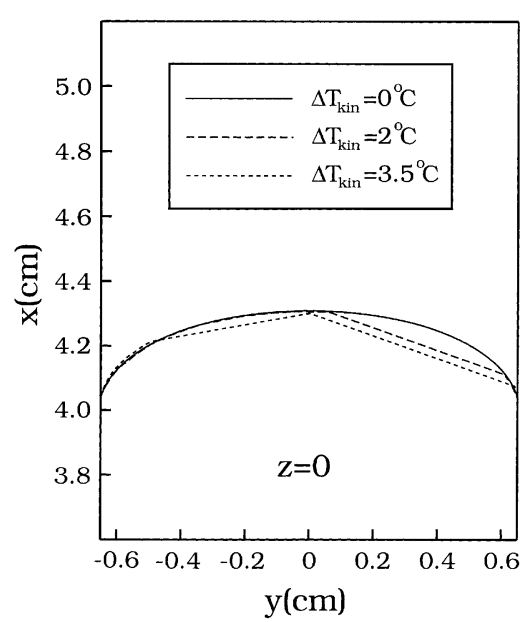

(b)

Fig. 3. Effect of supercooling on the interface shape: (a) at $z=0$; (b) at $y=0$.

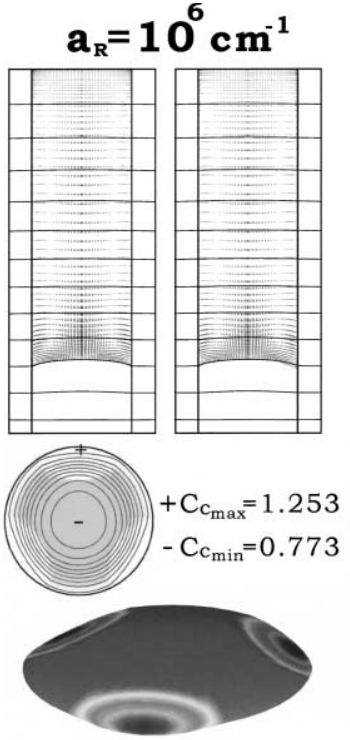

(a)

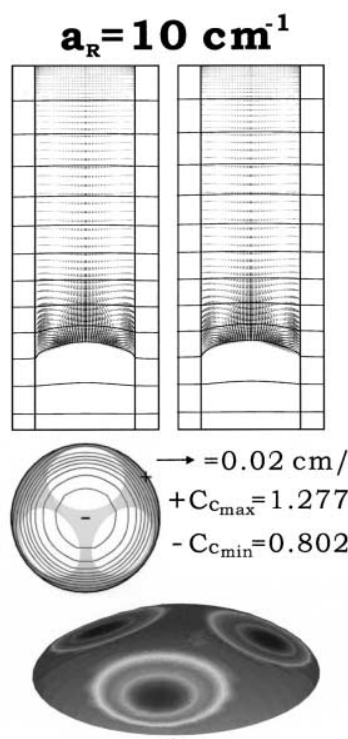

(b)

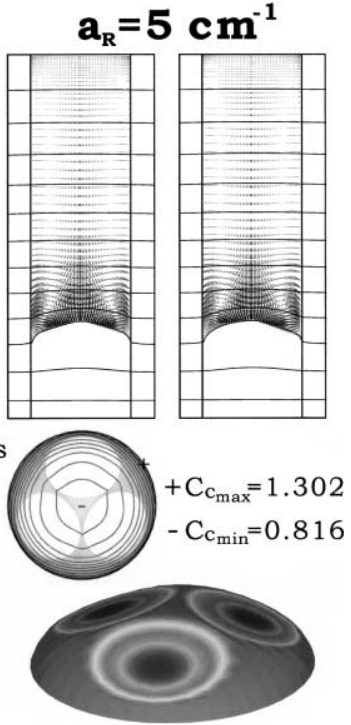

(c)

Fig. 4. Effects of absorption coefficient (internal radiation) in the crystal on the flow, thermal and dopant fields, and interface shape: (a) $a_{\mathrm{R}}=10^{6} \mathrm{~cm}^{-1}$; (b) $a_{\mathrm{R}}=10 \mathrm{~cm}^{-1}$; (c) $a_{\mathrm{R}}=5 \mathrm{~cm}^{-1} ; \Delta T_{\text {kin }}=2^{\circ} \mathrm{C}$. The grey level for temperature counters at the interface is the same as that in Fig. $2 b$.

indeed affects the faceting, both its position and size. Fig. 4 shows the effect of internal radiation in the crystal by reducing the Rosseland mean absorption coefficient $\left(a_{\mathrm{R}}\right)$. For the case of no internal radiation, the interface is only slightly convex as shown in Fig. 4a; this convexity is also due to the larger crystal thermal conductivity $\left(k_{\mathrm{c}}^{\text {cond }}=0.1 \mathrm{~W} \mathrm{~cm}^{-1} \mathrm{~K}^{-1}=2 k_{\mathrm{m}}\right)$. The convection 


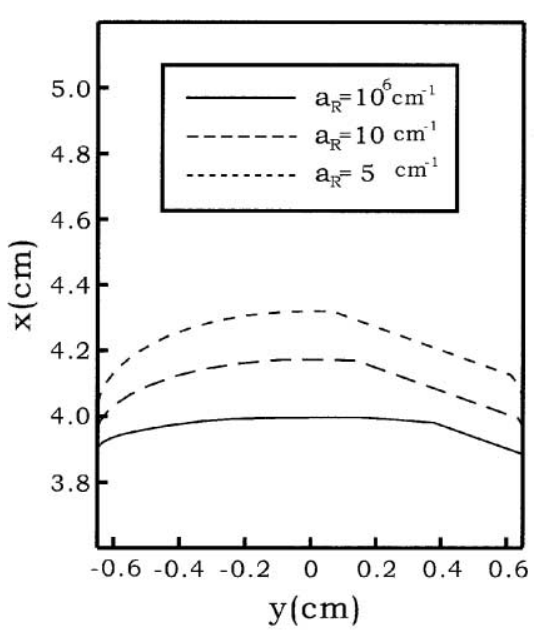

(a)

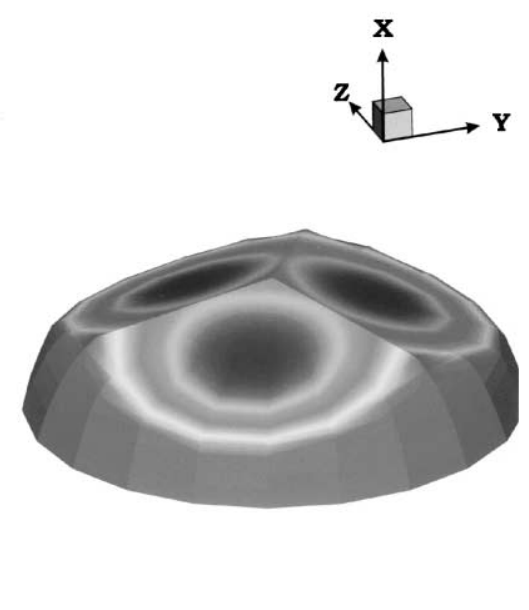

(b)

Fig. 5. (a) Effect of internal radiation on the interface shape; (b) an interface shape obtained from $a_{\mathrm{R}}=1 \mathrm{~cm}^{-1}$ and $d=0.25 \mathrm{~cm}$; $\Delta T_{\text {kin }}=2^{\circ} \mathrm{C}$.

due to the small interface deformation is much weaker as well. With $2^{\circ} \mathrm{C}$ supercooling, the facets appear at the edge of the interface (bottom plot of Fig. 4a). Because the facets have a larger slope than that of the rough surface, the facet edges are lower than the rim of the rough surface. As a result, the radial segregation increases (smaller $C c_{\min }$ ) due to the dopant accumulation there. Reducing the absorption coefficient, or increasing internal radiation, increases the interface convexity, as shown in Figs. $4 \mathrm{~b}$ and c. Besides the stronger flow, the facets move inwards. Again, if one further increases the interface convexity, the facets will appear around the center of the interface, and singular edges may appear, which is quite typical in experiments [5]. We also put the interfaces of Fig. 4 together for comparison in Fig. 5a. A faceting of a highly convex interface is shown in Fig. 5b; $a_{\mathrm{R}}=1 \mathrm{~cm}^{-1}$. In this case, the three facets join forming three singular edges. Again, if the supercooling is reduced, e.g., by reducing the growth rate, the area of faceting can be reduced. However, they may still remain in the same pattern. As a result, the crystal quality is significantly affected. Because the Rosseland approximation is not suitable for the case with a thin optical distance, the result shown in Fig. $5 \mathrm{~b}$ is only used as a reference.

\subsection{Effect of convection}

The oxide melt is usually very viscous and the Bridgman configuration is thermally stable. Therefore, as we have illustrated so far, the convection in the melt is very weak. As a result, the thermal fields and also the interface morphology are not much affected. In order to see the effect of melt flow, we have purposely reduced the melt viscosity, and the result is shown in Fig. 6b; the result of Fig. $2 b$ is placed in Fig. 6a for comparison. Although the melt convection is significantly enhanced, the effect of melt flow on the thermal fields, and also the interface, is still small. Dopant segregation is influenced more. Due to the better mixing, the segregation is reduced by the stronger flow shown in Fig. 6b. The cusps in the isoconcentration lines at the interface almost disappear.

To further increase the effect of flow on the thermal fields, we tilt the ampoule at $45^{\circ}$, as shown in Fig. 6c, for comparison. Now, due to the much stronger convection, the isotherms are much distorted. As a result, the interface becomes much more asymmetric, seen from the $x-y$ plane; 


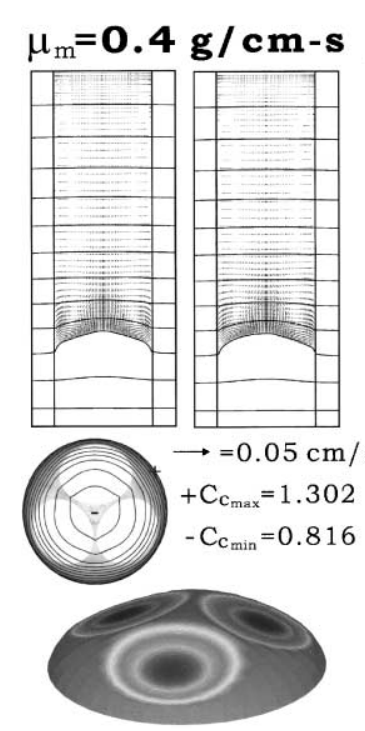

(a)

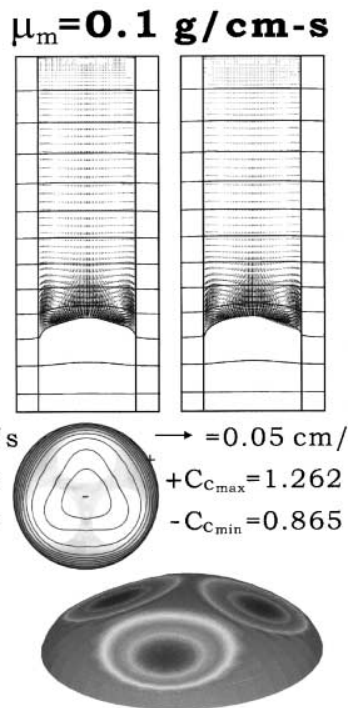

(b)

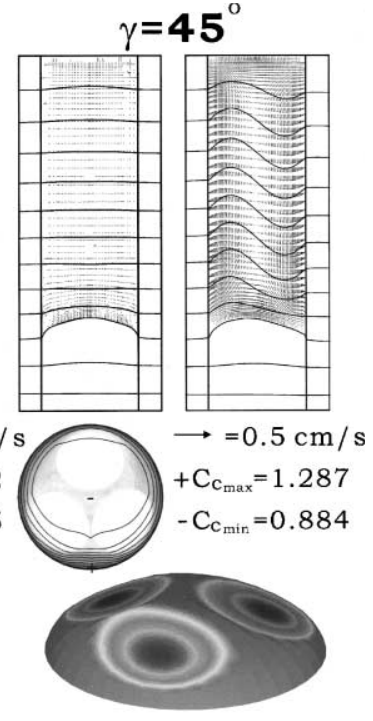

(c)

Fig. 6. Effect of convection on the dopant field and interface shape: (a) $\mu_{\mathrm{m}}=0.4 \mathrm{~g} \mathrm{~cm}^{-1} \mathrm{~s}^{-1}$; (b) $\mu_{\mathrm{m}}=0.1 \mathrm{~g} \mathrm{~cm}^{-1} \mathrm{~s}^{-1}$; (c) $\mu_{\mathrm{m}}=$ $0.4 \mathrm{~g} \mathrm{~cm}^{-1} \mathrm{~s}^{-1}$ and $\gamma=45^{\circ} ; \Delta T_{\text {kin }}=2^{\circ} \mathrm{C}$. The grey level for temperature counters at the interface is the same as that in Fig. $2 \mathrm{~b}$.

the reference velocity scale is enlarged 10 times for comparison. Due to the interface deformation by the flow, two facets join forming a singular edge, but the other remains separated. Although the stress is not calculated here, the stress concentration due to the singular edge can be anticipated.

\subsection{Effect of thermal gradients}

As shown by Eq. (14), the facet area should be reduced with the increasing axial thermal gradient. In fact, this is a typical way in practice to reduce faceting. To illustrate that, we use two linear heating profiles for comparison; one has a thermal gradient of $25 \mathrm{~K} \mathrm{~cm}^{-1}$ (Fig. 7a), and the other $200 \mathrm{~K} \mathrm{~cm}^{-1}$ (Fig. 7b). The calculated results are shown in Fig. 7 for $2{ }^{\circ} \mathrm{C}$ supercooling; $a_{\mathrm{R}}=$ $5 \mathrm{~cm}^{-1}$. With a higher thermal gradient, for $200 \mathrm{~K} \mathrm{~cm}^{-1}$, the convection is apparently stronger. As a result, its dopant distribution is more uniform. The facet area shown at the bottom plot is clearly reduced for the high thermal gradient. In fact, if we plot the facet size versus the square root of thermal gradient, the relation is consistent with the simple theory, as shown in Fig. 8. In fact, if the local thermal gradient at the facet center $\left(G_{\text {inner }}\right)$ is used, the result is even better. The result based on Eq. (14) is also plotted for comparison. However, it seems to be overpredicted, because the radius of curvature at the interface is not uniform. Using the inner thermal gradient for Eq. (14) does not improve the prediction much. In this case (with a linear heating profile), the inner and outer thermal gradients, though not the same, do not differ much. Nevertheless, the difference increases rapidly if the heating profile deviates from the linear one. Therefore, for such a case, the detailed thermal modeling becomes important for getting an accurate local thermal gradient in predicting the facet size.

\subsection{Abnormal segregation}

It is well known that the segregation on facets occurs in a nonequilibrium manner and the segregation coefficient $K$ can differ dramatically from that on a rough surface $[19,20]$. For semiconductor growth, the famous example is the growth of Te-doped InSb by the Czochralski 


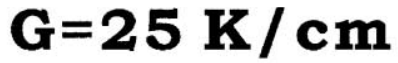

\section{$\mathbf{G}=\mathbf{2 0 0} \mathrm{K} / \mathrm{cm}$}
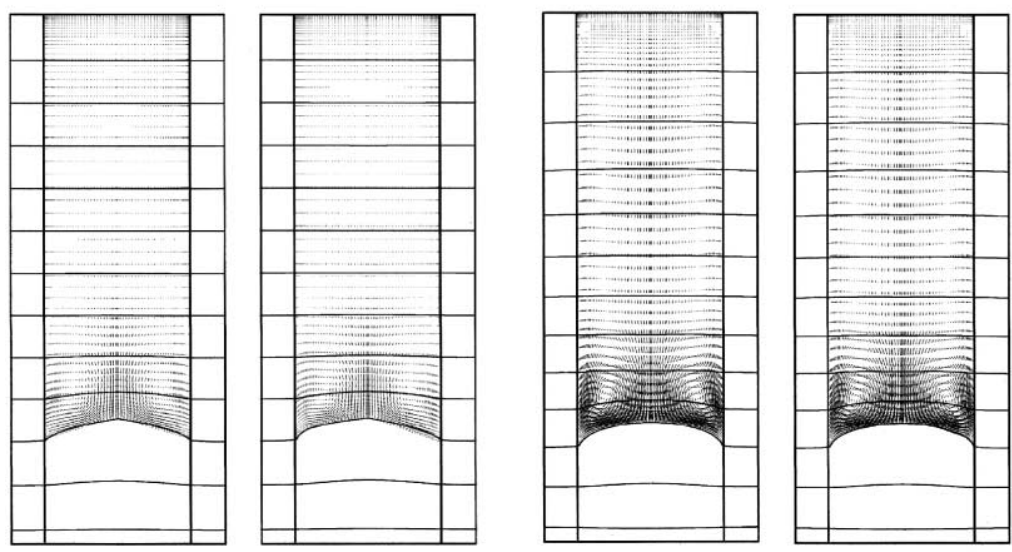

$\longrightarrow=0.03 \mathrm{~cm} / \mathrm{s}$

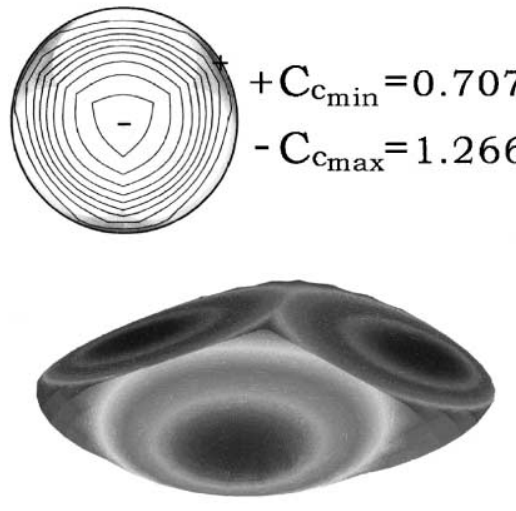

(a)
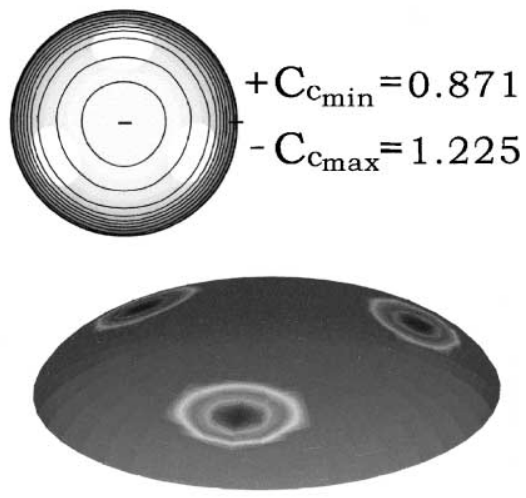

(b)

Fig. 7. Effect of thermal gradient of the heating profile on the flow, dopant fields, and interface shape: (a) $G=25 \mathrm{~K} \mathrm{~cm}^{-1}$; (b) $G=200 \mathrm{~K} \mathrm{~cm}^{-1}$; the heating profile is linear. $\Delta T_{\text {kin }}=2^{\circ} \mathrm{C}$ and $a_{\mathrm{R}}=5 \mathrm{~cm}^{-1}$. The grey level for temperature counters at the interface is the same as that in Fig. $2 b$.

method, where Te-rich InSb, corresponding to a higher segregation coefficient, appears on the $\left\{\begin{array}{lll}1 & 1 & 1\end{array}\right\}$ facet [19]. In fact, for the growth of BSO, the preferential substitution of $\mathrm{Bi}^{3+}$ ion for $\mathrm{Si}^{4+}$ ion in the $\left\{\begin{array}{lll}1 & 0 & 0\end{array}\right\}$ or $\left\{\begin{array}{lll}1 & 1 & 0\end{array}\right\}$ facet has let to a dark core (near the green-blue region) in the grown crystal [6]. Therefore, to illustrate the abnormal segregation, we allow the segregation coefficient at the facets $\left(K_{\mathrm{f}}\right)$ to be different. Fig. 9 shows the effects of $K_{\mathrm{f}}$, while the coefficient $K$ at the rough surface remains the same being 0.1 here.
The $K_{\mathrm{f}}$ value is $0.05,0.1$, and 0.2 in Figs. $9 \mathrm{a}, \mathrm{b}$, and $\mathrm{c}$, respectively. As shown, the overall dopant distribution in the melt is not altered much by varying the coefficient, except the absolute value. In fact, from the boundary condition, Eq. (9), one may be aware of the difference in the factor $(1-$ $K$ ) being $0.8-0.95$. Nevertheless, near the interface, the dopant concentration is affected significantly by the $K_{\mathrm{f}}$ value, especially, the concentration in the grown crystal $(K C)$. Because the solid state diffusion is neglected here, the dopant concentra- 


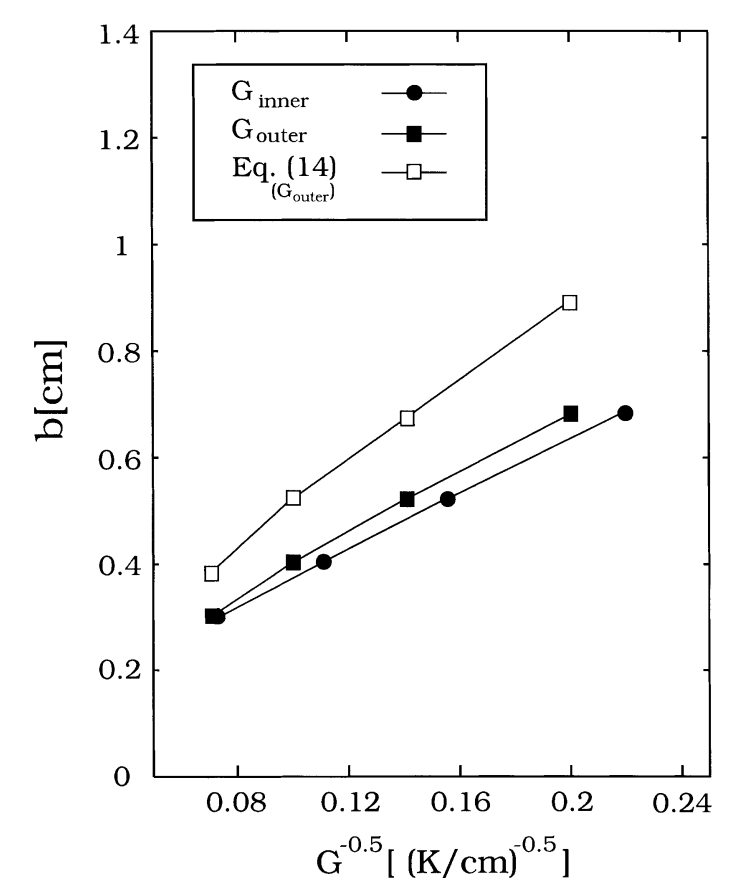

Fig. 8. Facet size (b) as a function of the square root of thermal gradient $\left(G^{0.5}\right)$; the inner gradient $\left(G_{\text {inner }}\right)$ is obtained locally at the facet center, while $G_{\text {outer }}$ is the gradient of the heating profile. The result for Eq. (14) is based on the outer thermal gradient.

tion is not continuously varied from the rough surface to the facets, even though the solute field is continuous in the melt. With a smaller $K_{\mathrm{f}}$, the dopant concentration inside the facets is significantly lower, as shown in Fig. 9a. On the contrary, with a higher $K_{\mathrm{f}}$, the dopant content inside the facets significantly increases, as illustrated in Fig. 9c. Due to the flow, the highest concentration appears at the outer edge of the facets. The dark core situation found in BSO growth is a typical example of the much larger segregation for $\mathrm{Bi}^{3+}$ ions inside the $\left\{\begin{array}{lll}1 & 0 & 0\end{array}\right\}$ or $\left\{\begin{array}{lll}1 & 1 & 0\end{array}\right\}$ facets. In addition, Cockayne [2] also discussed anomalous segregation of oxygen due to faceting, which is probably the cause of the strained core in Czochralski growth of YAG crystals.

Furthermore, for high doping situation, the dopant content may also affect supercooling. In such a case, two-way coupling needs to be considered. In this report, the dopant concentration is assumed to be low so that the heat flow and the interface shape are not affected.

\section{Conclusions}

In this study, we perform for the first time a 3D simulation of facet formation and its coupled flow and dopant segregation using a finite volume method. Although we consider here only the $\{211\}$ facets, the approach can be extended to other facet planes, and multiple facet families as well. Interestingly, even though the facets break axisymmetry, the heat transfer and melt flow are pretty much symmetric, except the dopant distribution near the interface. The growth at different growth directions may introduce higher asymmetry that may generate a clearer 3D flow. On the other hand, the highly asymmetric faceting is not preferred in crystal growth.

The kinetic data, the relationship between growth rate and supercooling, are important for the modeling, but these data are scattered. Since the mechanisms for facet formation are extremely important, microscopic modeling may be incorporated into the model. Once the kinetic information is known, it can be implemented easily into the heat flow model providing a more realistic simulation of oxide growth.

The size and position of the facets, as well as the formation of singular edges, are strongly affected by the interface shape and thus by the heat transfer. Hence, a more accurate model for internal radiation in the crystal (may be in the melt as well) is necessary for a better prediction. In this report, the Rosseland diffusive model is used, and it is suitable only for optically thick materials. For materials with a small optical thickness, a more rigorous model should be used. Again, for 3D simulation, a better model requires a much higher computing cost. For engineering applications, a tradeoff is to use the $P 1$ approximation [14] by solving an additional differential equation for radiosity. Besides the numerical simulation, accurate data for thermal radiation and interfacial kinetics are inevitable for a quantitative prediction of facet formation. 


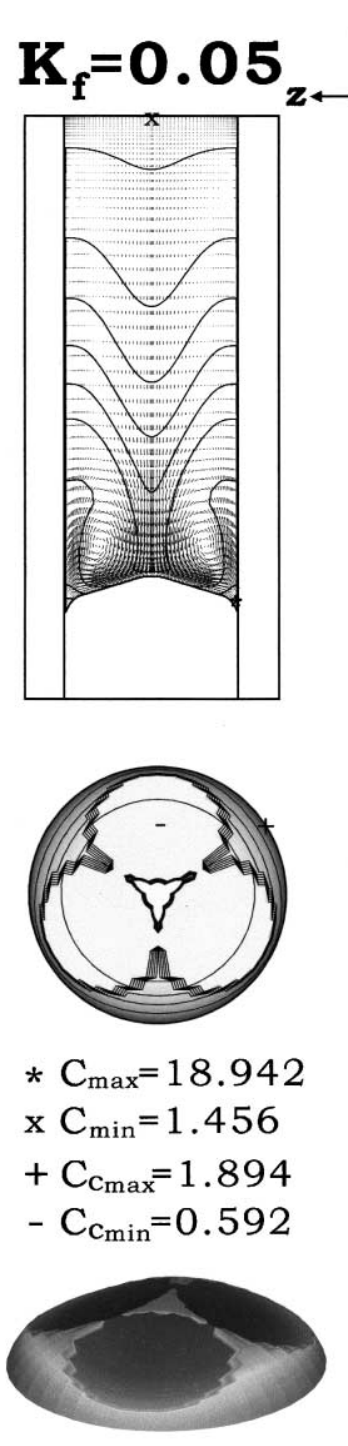

(a)
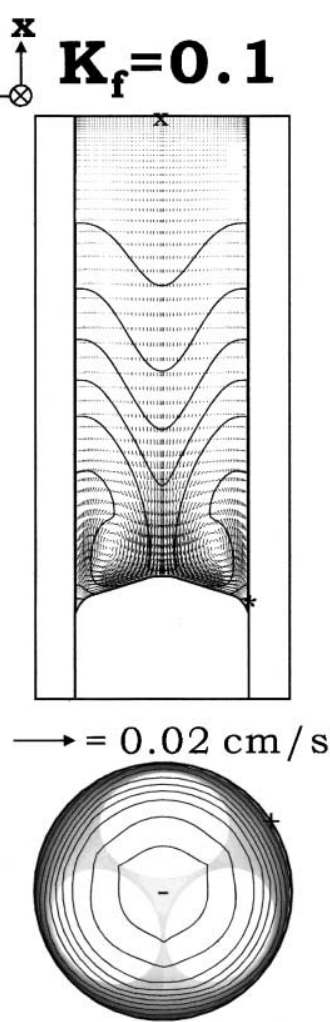

* $\mathrm{C}_{\max }=13.302$

$\mathrm{x} \mathrm{C}_{\text {min }}=1.302$

$+\mathrm{C}_{\mathrm{c}_{\max }}=1.302$

$-\mathrm{C}_{\mathrm{c}_{\text {min }}}=0.816$

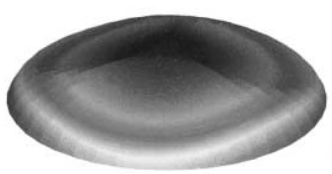

(b)

\section{$\mathbf{K}_{\mathrm{f}}=\mathbf{0 . 2}$}
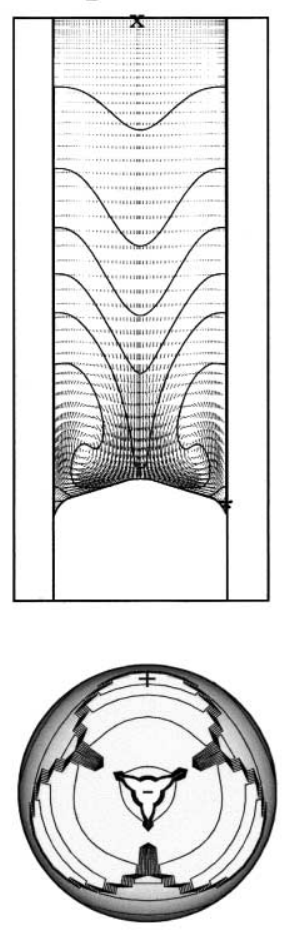

* $\mathrm{C}_{\max }=8.043$

$\mathrm{x}_{\text {min }}=1.172$

$+\mathrm{C}_{\mathrm{C}_{\max }}=1.389$

$-\mathrm{C}_{\mathrm{c}_{\min }}=0.511$

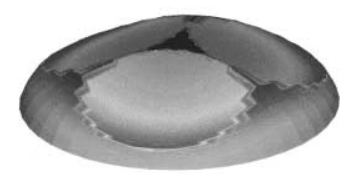

(c)

Fig. 9. Effect of different segregation coefficient at the facet $\left(K_{\mathrm{f}}\right)$ on the dopant segregation: (a) $K_{\mathrm{f}}=0.05$; (b) $K_{\mathrm{f}}=0.1$; (c) $K_{\mathrm{f}}=0.2$; $K=0.1, \Delta T_{\text {kin }}=2{ }^{\circ} \mathrm{C}$, and $a_{\mathrm{R}}=5 \mathrm{~cm}^{-1}$. The grey counters are for concentration at the interface; the medium grey is for $C_{\mathrm{C} \text { max }}$ and dark grey for the $C_{\mathrm{C} \min }$.

\section{Acknowledgements}

The authors are grateful for the support from the National Science Council and the National Center for High Performance Computing of the Republic of China under Grant No. 89-2214-E002-040. Valuable discussion with Prof. N.B. Ming of Nanjing University is highly appreciated. CWL also acknowledges Profs. S. Brandon and P. Ramasamy for careful reading of the manuscript and fruitful discussion. Especially, the Frank's paper provided by Prof. D.T.J. Hurle regarding the stress concentration at singular edges is also appreciated. 


\section{References}

[1] J.C. Brice, J. Crystal Growth 6 (1970) 205.

[2] B. Cockayne, J. Crystal Growth 42 (1977) 413.

[3] D.T.J. Hurle, Crystal Pulling from the Melt, Springer, Berlin, 1993, p. 51.

[4] A.G. Petrosyan, Kh.S. Bagdasarov, J. Crystal Growth 34 (1976) 110.

[5] N.B. Ming, Y.S. Yang, Acta Phys. Sin. 28 (1979) 285 (in Chinese).

[6] X. Xu, J. Liao, B. Shen, P. Chao, X. Chen, C. He, J. Crystal Growth 133 (1993) 267.

[7] D.T.J. Hurle, The present and potential impact of modeling in crystal growth, Third International Workshop on Modeling in Crystal Growth, October 18-20, 2000, Stony Brook, New York, USA.

[8] Y. Liu, A. Virozub, S. Brandon, J. Crystal Growth 205 (1999) 333.

[9] S. Brandon, A. Virozub, Y. Liu, in: H.J. Scheel, T. Fukuda (Eds.), Technology of Crystal Growth and Epitaxy, Wiley, New York, to appear.
[10] P.M. Adornato, R.A. Brown, J. Crystal Growth 80 (1987) 155.

[11] S. Brandon, J.J. Derby, J. Crystal Growth 121 (1992) 473.

[12] D. Vizman, I. Nicoara, D. Nicoara, J. Crystal Growth 169 (1996) 161.

[13] D. Vizman, I. Nicoara, G. Müller, J. Crystal Growth 212 (2000) 334.

[14] M.F. Modest, Radiative Heat Transfer, McGraw-Hill, New York, 1993, p. 487.

[15] V.V. Voronkov, Krystallografiya 17 (1972) 909.

[16] C.W. Lan, M.C. Liang, J. Comp. Phys. 152 (1999) 55.

[17] V.J. Fratello, C.D. Brandle, J. Crystal Growth 128 (1993) 1006.

[18] W. Bardsley, F.C. Frank, G.W. Green, D.T.J. Hurle, J. Crystal Growth 23 (1974) 341.

[19] J.B. Mullin, K.F. Hulme, J. Phys. Chem. Solid 17 (1960) 1 .

[20] J.B. Mullin, in: R.J. Malik (Ed.), III =V Semiconductor Materials and Devices, Elsevier, Amsterdam, 1987, p. 3. 